'Departamento de Medicina Intensiva. Facultad de Medicina Pontificia Universidad Católica de Chile. Santiago, Chile. Instituto de Ingeniería Matemática y Computacional, Escuela de Ingeniería, Pontificia Universidad Católica de Chile. Santiago, Chile. a MSC FACP.

${ }^{\mathrm{b}}$ Residente de Medicina Intensiva. Ingeniero Civil Industrial.

Los autores declaran no tener conflictos de interés. Trabajo no recibió financiamiento.

Enviado el 2 de junio de 2020, aprobado el 5 de junio de 2020 .

Correspondencia a: Dr. Max Andresen H MsC FACP Departamento de Medicina Intensiva, Facultad de Medicina, Pontificia Universidad Católica de Chile.

Diagonal Paraguay 362. Santiago, Chile. andresen@med.puc.cl

\section{La criticidad de las Unidades de Intensivo. Ampliando las Unidades en tiempos de pandemia}

\author{
MAX ANDRESEN ${ }^{1, \mathrm{a}}$, PABLO BORN ${ }^{1, \mathrm{~b}}$, EDUARDO KATTAN ${ }^{2}$, \\ MAGDALENA VERA ${ }^{1}$, ALEJANDRO CATALDO ${ }^{2, c}$, \\ CAROLINA RUIZ ${ }^{1}$ SEBASTIÁN BRAVO ${ }^{1}$
}

\section{Expanding ICU facilities in a pandemic scenario}

Our country is suffering the effects of the ongoing pandemic of coronavirus disease (COVID-19). Because the vulnerability of healthcare systems, especially the intensive care areas they can rapidly be overloaded. That challenge the ICUs simultaneously on multiple fronts making urgent to increase the number of beds, without lowering the standards of care. The purpose of this article is to discuss some aspects of the national situation and to provide recommendations on the organizational management of intensive care units such as isolation protocols, surge in ICU bed capacity, ensure adequate supplies, protect and train healthcare workers maintaining quality clinical management.

(Rev Med Chile 2020; 148: 674-683)

Key words: Intensive Care Units; Respiratory Distress Syndrome; SARS Virus.

\begin{abstract}
A ctualmente, nuestro país está enfrentando la pandemia de síndrome respiratorio agudo grave por coronavirus 2 (SARS-CoV-2), la cual ha causado hasta la fecha a nivel mundial más de 350.000 fallecidos y 6 millones de personas infectadas. Esta gravísima situación ha estresado al máximo los sistemas de Salud a nivel mundial. Para lograr mitigar el impacto de esta emergencia sanitaria se debe involucrar a la sociedad en su conjunto. Así, el Gobierno Central define las políticas de salud a implementar y los sistemas de salud se deben adaptar a la contingencia con prontitud y flexibilidad, aumentando la capacidad de atención. Sin embargo, es clave el cumplimiento, por parte de la población, de las medidas sanitarias dictadas por las autoridades, tanto en el autocuidado como en el cumplimiento de cuarentenas y confinamiento. Todas son esenciales, pero esta última representa una mayor complejidad, debido a aristas sociales, económicas y culturales de la población. Dada la alta contagiosidad del virus
\end{abstract}

aparecen focos en los estratos de mayor vulnerabilidad, en donde el grado de hacinamiento es mayor, por lo que no es siempre posible realizar un aislamiento efectivo. Lo anterior no es el foco a discutir en esta comunicación, pero es sin duda es un elemento central a considerar.

En este artículo discutiremos algunas medidas que se toman en las Unidades de Pacientes Críticos para poder responder al exceso de demanda en tiempos de crisis.

Cada país, región, ciudad y Unidad de Cuidados Intensivos tienen realidades y desafíos particulares $^{1,2}$, por lo tanto, la política para desarrollar el incremento de camas y que sistema a emplear, en una situación de crisis, es particular en cada contexto.

De esta forma no es comparable la política de expansión que tenga un hospital de referencia respecto al de un hospital de una ciudad alejada con una población reducida, o la política macro de país, en donde influyen múltiples variables. 


\section{En Sudamérica sabíamos que iba llegar esta pandemia. Aún no sabemos cuánto va a durar ni el número afectados posibles}

Así, ciertos gobiernos tomaron una política más bien expectante y no activa ${ }^{3}$, discordante con las sugerencias entregadas por entes técnicos y de salud pública, discrepancias lamentables en escenarios adversos y complejos. Así, tanto la magnitud de crecimiento en recursos para la atención, como la oportunidad de llevarlo a cabo, variará en cada país según las políticas públicas y la política sanitaria.

En esta epidemia pasamos de una situación convencional, caracterizada por cuidados críticos habituales, a una etapa de contingencia; es en esta etapa en que se han de adaptar áreas para manejar pacientes críticos diferentes a la que usamos en la práctica habitual. En este momento debemos aumentar nuestra planta de personal sanitario y de espacios para mantener los mismos estándares de atención a la de una situación convencional. Distinta es la situación de crisis, acá los sistemas de salud están sobre exigidos y se produce atención de pacientes muchas veces en áreas menos seguras con equipos menos entrenados, y en la cual muchas veces faltan suministros clínicos esenciales ${ }^{4}$. Si bien esta situación no es deseable, la experiencia mundial nos muestra que ha ocurrido incluso en países de altos recursos.

Lo anterior se genera por un excesivo número de pacientes que requieren atención crítica inmediata. Esto se produjo al comienzo de la pandemia, particularmente en China, por el desconocimiento de la contagiosidad y severidad de los pacientes que presentaban COVID-19. Asimismo, ocurrió semanas después en Europa (Italia, España y Reino Unido) y en EE. UU. (Estado de Nueva York).

Es clave para una correcta planificación evaluar cuáles son nuestras fortalezas y oportunidades de mejora, siendo lo más objetivos posibles, mediante un análisis de vulnerabilidad (Figura 1). Conocer cuan bien estamos preparados, respecto de recursos técnicos humanos y organizacionales, conocer el impacto que se viene, nuestra capacidad para responder y las capacidades reales de expansión de las unidades de cuidados intensivos ${ }^{5}$. Esto permitirá desarrollar nuestra planificación para enfrentar la crisis.

La ventaja que tuvimos en Sudamérica fue el desfase temporal. La epidemia de COVID-19 fue declarada por la OMS como una emergencia de salud pública de preocupación internacional el 30 de enero de 2020. Así, pudimos contar con aproximadamente ocho semanas para poder preparar$n^{n}{ }^{6}$. Ya contábamos con información asociada al tipo de pacientes y sus comorbilidades (ej.: edad, población de riego, mortalidad, transmisibilidad, terapias posibles) y algunos reportes del comportamiento clínico de estos, eso por lo menos del punto vista sanitario. Esta valiosa información nos entregaba nociones de los requerimientos de salud necesarios para enfrentar esta crisis. La principal desventaja fue que muchos de nuestros países cuentan con una precaria infraestructura de salud, altos niveles de pobreza y hacinamiento.

En nuestro país la pandemia se comportó inicialmente, como una situación relativamente estable, con una política de parte de la autoridad sanitaria de contención y aislamiento de los casos confirmados como sospechosos con medidas de confinamiento y restricción de movilidad social, con cuarentenas dinámicas en áreas de estrato socioeconómico medio alto. Esto permitió mantener una situación más bien estacionaria sin llegar a grandes peaks y sobredemandar al sistema, con ocupaciones de unidades de cuidados intensivos en promedio alrededor de $60 \%$ a $70 \%$, siendo $50 \%$ de los enfermos en ventilación mecánica por COVID-19 el resto por no COVID-197. Cuando se levantaron parcialmente esas medidas y la infección se propagó a zonas con poblaciones más

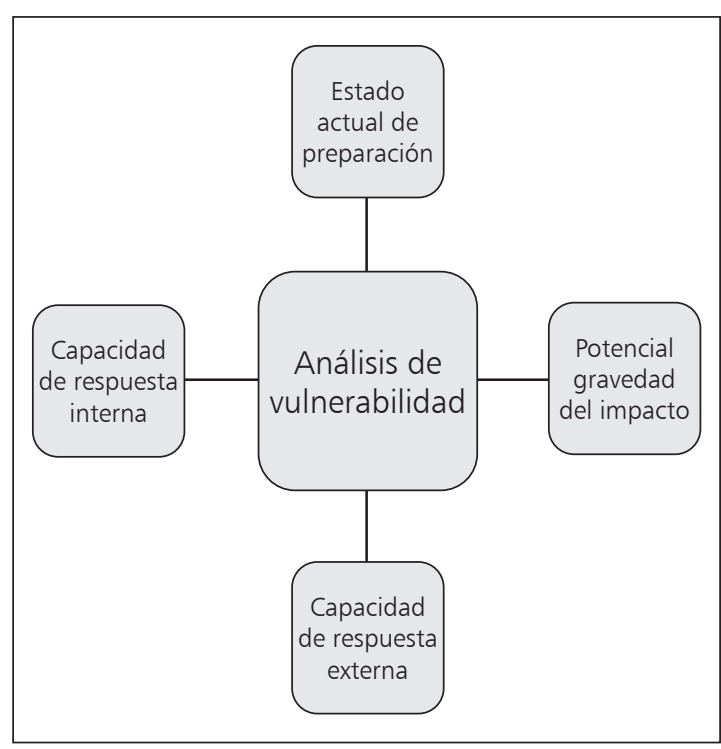

Figura 1. Análisis de vulnerabilidad. 
vulnerables y de mayor densidad poblacional, se evidenció un categórico cambio, traduciéndose en un alza de contagios y por ende mayor demanda asistencial en el sistema público y privado de salud. En la actualidad: ocupación UCI en región metropolitana 98\%, 87\% a nivel nacional, en cuarentena en varias ciudades, cordones sanitarios y progresando en niveles de testeo y trazabilidad con meta de completar expansión de al menos del doble de camas críticas respecto de las basales.

La evolución de la situación actual es y será evidentemente heterogénea tanto a nivel local como regional, ya que se producen algunas áreas de sobredemanda por aparición de focos no controlados. Esto ha generado requerimientos de traslado de pacientes, profesionales sanitarios y equipamiento, en particular ventiladores mecánicos hacia donde son más necesarios, ya que la política ministerial en Chile ha aglutinado los recursos a través de la Red centralizada COVID.

\section{¿Qué desafíos enfrentamos en las Unidades de Cuidados Intensivo para decidir la alocación de los recursos?}

Sabemos que la mayor parte de los enfermos son asintomáticos y que de los pacientes sintomáticos aproximadamente $5 \%$ va a llegar a Unidades de Pacientes Críticos ${ }^{8}$, por lo tanto, ese es el referente que debemos tener para poder adaptar las necesidades país, regionales y locales.

Por otra parte, de los pacientes hospitalizados entre $25 \%$ al $30 \%$ de ellos va a requerir $\mathrm{UCI}^{9,10}$. En el escenario de pacientes críticos, en una serie de 52 pacientes en UCIs de Wuhan se observó una sobrevida de $50 \%$ al día 25 de seguimiento ${ }^{11}$. Por otro lado, una serie de 257 pacientes críticos ingresados en Nueva York describe una mortalidad intrahospitalaria acumulada de 39\% a los 40 días de seguimiento ${ }^{12}$.

Tenemos además grupos de riesgo claramente definidos, con un quiebre en la sobrevida a partir de los 65 años $^{13}$, situación que se repitió en otros países. Así en China mortalidad de $38 \%$ en pacientes en cuidados críticos, en Italia (región de Lombardía) fallecimientos en cuidados intensivos de $26 \%$, con mortalidad de $30 \%$ en pacientes sobre 60 años y $40 \%$ sobre los 70 años ${ }^{14}$.

Por lo tanto, esa era la condición que debíamos enfrentar como crisis: alto volumen de pacientes graves y con elevada mortalidad, hospitalizados que ocuparan entre $25 \%$ a $30 \%$ de la cama de intensivos, grupos de riesgo claramente definidos ecuación complejizado por estadías prolongadas en ventilación mecánica (2-3 semanas).

En la etapa previa a la crisis debemos desarrollar los planes de contingencia; en Sudamérica, como comentamos, tuvimos alrededor de seis a ocho semanas, por lo tanto, no fue particularmente larga. Esta es una etapa en general sin incidentes, un período relativamente calmo pero es una gran oportunidad para poder desarrollar el sistema, asegurar operaciones de suministros nacionales o extranjeros, generar coordinaciones nacionales, locales y a nivel hospitalario. De ahí que es de máxima importancia ya que partir de acá se definen las políticas y por ende del destino de nuestros pacientes.

Tenemos que definir en estas etapas cuáles serán las áreas de atención de nuestros pacientes (Figura 2), cuanto debemos aumentar el número de camas, definir los equipos médicos, en particular aquellos de urgencia, intensivo, intermedio y sala básica. Asimismo, generar una estrecha relación con los equipos de anestesiología, dejando en segundo plano áreas quirúrgicas, ya que esta actividad debe ser limitada solo para casos de urgencia real y aquellos tiempo dependiente, como los pacientes oncológicos.

Es central el suministro de productos farmacéuticos, insumos médicos, equipos de protección personal, y empezar a generar guías de criterios de admisión, egreso y de exclusión: por ejemplo, paciente gran quemado, mal pronóstico oncológico, daño pulmonar severo dependiente de oxígeno, etc.

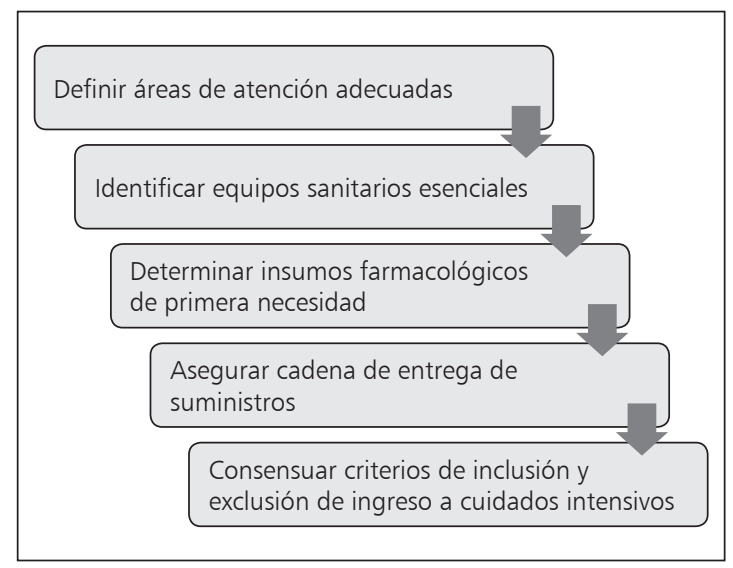

Figura 2. Aspectos centrales a definir en primera etapa. 


\section{La pregunta ahora es cuánto expandirse}

Como comentamos previamente las realidades son individuales. En el mundo se ha descrito este desafío tempranamente tras el inicio de casos locales de COVID-19 ${ }^{15}$.

La política más utilizada es el empleo progresivo de áreas reconvertidas. Así, luego de planificar nuevas áreas de cuidados intensivos, con el equipamiento suficiente, estas se empiezan a utilizar dependiendo de la etapa donde se encuentre la contingencia sanitaria, destinando los equipos de salud hacia esas áreas. El grado de calificación del equipo de salud va a ser dependiente de la demanda de atención, vale decir: inicialmente los pacientes serán admitidos y cuidados por equipos dedicados a los cuidados intensivos, y posteriormente con equipos capacitados o supervisados por especialistas de Intensivo ${ }^{16}$.

\section{¿Cuál cama ocuparemos?}

Inicialmente se priorizan las camas de la unidad de pacientes críticos incluyendo las de cuidados intermedios. Estas camas de intermedio se flexibilizan y se generan áreas de manejo de pacientes críticos; luego la sugerencia es utilizar área de unidades coronarias, intensivos pediátricos, luego unidad de manejo de postoperatorios, luego unidades de procedimientos como broncoscopía, endoscopía, etc. para en última instancia habilitar salas de hospitalización general dispuestas para manejo de pacientes más críticos ${ }^{17}$. Evidentemente esta secuencia implica, en lo posible, no bajar los estándares de atención, sin embargo, se resienten los grados de monitoreo y supervisión por especialistas.

Experiencias extranjeras en hospitales terciarios y privados, como en el caso de Australia, muestran que el incremento de camas dentro de la UCI, se puede lograr solo entre 5 a $20 \%$ aproximadamente. Sin embargo, cuando se utilizan áreas fuera de la UCI, se puede incluso triplicar la disponibilidad de cupos para el manejo de enfermo críticos.

Una encuesta en 175 de las 191 UCIs en Australia demostró un potencial de aumentar camas a $190 \%$ y ventiladores a $120 \%$, pero con requerimiento de aumento de personal a $250 \%{ }^{18}$.

En situación de saturación la sugerencia es la habilitación de hospitales de campaña, área donde las Fuerzas Armadas tienen mucha experiencia.

No debemos dejar pasar que la expansión es siempre limitada y por tanto el personal de salud debe administrar los recursos en forma juiciosa, desde los insumos básicos hasta generar los mejores protocolos médicos, por ejemplo: intubar bajo estrictos criterios y emplear métodos alternativos de oxigenación como la cánula nasal de alto flujo para lograr el menor números de ingresos a las Unidades más demandadas.

Estos nuevos espacios que se habilitan deben distribuirse en áreas separadas, una para el manejo exclusivo de pacientes con infección viral con equipos organizados y destinados solamente para este fin y otras áreas físicas para enfermos no COVID-19 ${ }^{19}$. En nuestro país la ocupación de camas críticas fue aproximadamente de 50\% para pacientes con COVID-19 y el otro 50\% para pacientes críticos habituales. Proporción que fue cambiando en la medida que progresó la pandemia. En nuestro país, actualmente $80 \%$ del total de camas críticas es ocupado por pacientes COVID-19 (+), la mayoría de ellos en ventilación mecánica invasiva ${ }^{7}$.

\section{El personal de salud}

El rol de los intensivistas es clave en la emergencia sanitaria, no se limita únicamente a ser considerados como tratantes, sino que además deben ser incluidos en el comité ejecutivo de los hospitales, generando y fortaleciendo comités internos dentro de las $\mathrm{UCI}^{20,21}$. Su rol es crucial en la educación de médicos que no trabajan habitualmente en estas unidades, al igual que la supervisión cuando la demanda de pacientes aumenta, tanto en la realización de procedimientos como en la toma de decisiones complejas ${ }^{22,23}$.

Los apoyos generalmente son otorgados por médicos de medicina interna, anestesiología y especialidades afines, pero también debemos integrar a médicos de especialidades más lejanas a labores de gestión, coordinación y administrativas, siempre importantes y que demandan mucho tiempo. También tenemos un rol fuera de la UCI cuando destinamos pacientes en unidades como coronaria, unidades de recuperación, etc., por lo tanto, el rol es extremadamente importante y central.

Se debe planificar muy bien el sistema de turnos $^{24}$. Normalmente los turnos cambian cada 12 horas; sin embargo, en casos de epidemia sea por 
la sobrecarga clínica, incomodidad en la actividad diaria (uso de elementos de protección personal, más la dificultad de acceder a instalaciones como alimentación, baño, etc.), se sugieren turnos cada ocho horas incluso cada seis horas. También cambia la proporción de pacientes a atender, lo habitual es de ocho pacientes por médico, aumenta generalmente a 10-12, incluso más cuando se privilegia la supervisión. Precaución con los turnos de noche donde baja la dotación incluso a 50\%. Por lo anterior se sugiere que exámenes y procedimientos se realicen en realicen horarios diurnos $^{25}$. Muchos centros proteger a médicos manteniéndolo un sistema On/Off, trabajando una semana y estando fuera otra semana.

Pensamos que el rol de la telemedicina ha cambiado mucho nuestra forma de trabajo y puede ser muy útil para el apoyo a distancia de UCI pequeñas por centros más complejos ${ }^{20}$.

Respecto al equipo de enfermería de UCI, su rol es clave en las estrategias de dotación de personal de la unidad, definiendo los equipos de turno, duración de los turnos y lograr mantener una dotación lo más estable posible ${ }^{19}$. Esto dado que con alta probabilidad se generara ausentismo por infecciones del personal de salud. Enfermería participa fuertemente en la capacitación de otras enfermeras que no son parte regular de la UCI, ej. Enfermeras de sala para manejar enfermos de mediana complejidad y expandir enfermería avanzada hacia aquellas profesionales que ya tienen conocimientos de cuidado críticos como enfermeras de postoperados y de pabellón. Es clave enfermería en el diseño del proceso de circulación de los pacientes para su admisión, implementación estricta de medidas de aislamiento y de su correcto uso mediante pautas de cotejo, movilización de casos sospechoso y confirmados, como también de la regulación de las visitas dentro de la unidad ${ }^{19,24}$.

Las enfermeras además deben reasignar la dotación en sistema de turnos, combinando profesionales de mayor y menor experiencia, asignando el número de pacientes por enfermera; se sugiere una enfermera cada 4 a 6 pacientes de gravedad leve a moderada; en UCI una enfermera cada dos a tres pacientes, debiendo ser $1 / 1$ en pacientes con ventilación mecánica en decúbito prono asociado a inestabilidad hemodinámica o en $\mathrm{ECMO}^{26}$.

Para poder mantener el personal de salud en buenas condiciones, sin ser afectados por la enfermedad, es esencial la capacitación en el correcto empleo de elementos de protección personal y la definición de los procedimientos de más alto riesgo (Tabla 1) para tomar las medidas necesarias ${ }^{27}$. Esto dado la alta transmisibilidad de la enfermedad.

La atención de estos pacientes debe reglarse para tratar de ingresar a los box lo menos posible de ahí que se regulan los procedimientos como: manejo de bombas fuera del box, visualización de pantallas de ventiladores, cambios de posición (en particular en pacientes en prono) frecuencia y forma de control de signos vitales, aspiraciones, diuresis, rotaciones de tubos, aspiraciones, etc.

Cuando tenemos personal que no está al corriente de todas las prácticas de UCI, debemos generar guías, pero esto no siempre es suficiente: debemos capacitar muy bien, implementarlas de buena forma y para ver el grado de cumplimiento de estos procedimientos emplear pautas específi$\operatorname{cas}^{28}$ (Figura 3).

En Estados Unidos, se ha desarrollado e impulsado la formación de enfermeras con altos estándares técnicos, conocidos como ICU Nurse Practitioner. Se les permite legalmente manejar pacientes críticos con altos grados de autonomía, realizar procedimientos más invasivos ( $\mathrm{p}$ ej., instalación de accesos venosos centrales), dejar indicaciones, coordinar evaluaciones por especialistas,

Tabla 1. Procedimientos con alto riesgo potencial de transmisión

- Humidificación de aerosoles
- Ventilación con bolsa-máscara
- Broncoscopía
- Reanimación cardiopulmonar
- Desconexión de tubo endotraqueal del ventilador
- Extubación
- Oxigenoterapia de alto flujo
- Ventilación oscilatoria de alta frecuencia
- Intubación
- Hisopados nasofaríngeos
- Aspiración nasotraqueal u orotraqueal
- Nebulización de medicamentos
- Ventilación de presión positiva no invasiva
- Traqueostomías




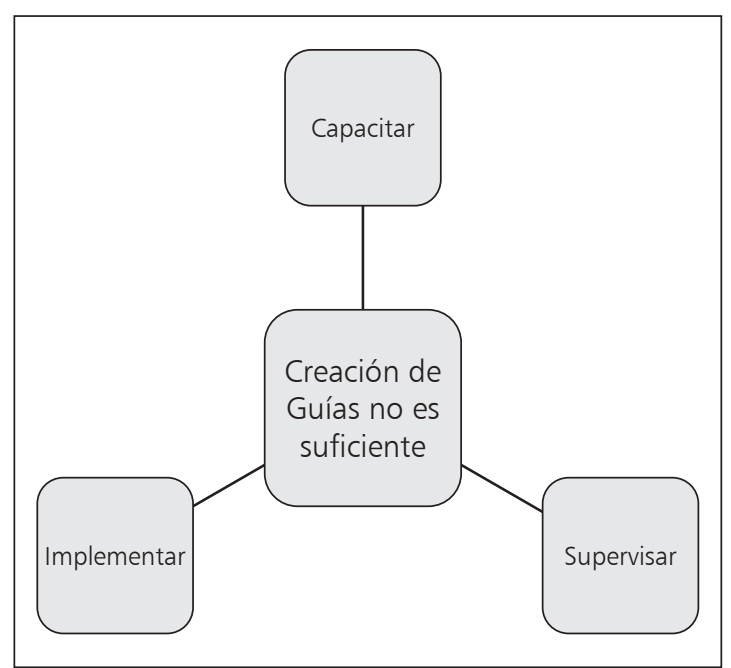

Figura 3. Proceso formativo para el equipo de salud.

etc. En Chile, nuestra enfermería de pacientes críticos es de excelencia, con muchas instancias de formación y postgrados, pero no existe este grado de especialización en particular.

Lo anterior lo comentamos como una alternativa posible futura de ampliación de personal con competencias cuando los especialistas médicos son escasos.

Los kinesiólogos y terapistas respiratorios toman cada vez más relevancia como apoyo en los cuidados y conducción de los pacientes en ventilación mecánica. Durante esta crisis, no solamente deben apoyar en el manejo ventilatorio sino también deben participar en la educación y manejo en áreas fuera de la unidad de críticos.
Dada la sobrecarga de trabajo es muy importante en situación de crisis el planificar los exámenes para optimizar los tiempos del personal y no sobrecargar los laboratorios y servicios de radiología (Figura 4 ).

Las autoridades hospitalarias deben estar conscientes de los elementos claves y más sensibles para el correcto funcionamiento de la UCI. Así, es fundamental listar los insumos médicos prioritarios y tenerlos disponibles en suficiente cantidad (Tabla 2). Estos son: antimicrobianos, coloides y cristaloides, agentes de sedación y bloqueo neuromuscular, drogas vaso activas, heparinas. Tema aparte es un buen funcionamiento del banco sangre que en este período si disminuye francamente su stock y deben hacerse campañas preventivas ${ }^{20,29}$.

El uso de ventiladores mecánicos es evidentemente clave en epidemias que afectan el sistema respiratorio; por el costo y dificultad de compra se puede generar quiebre de stock ${ }^{30}$. Se sugiere centralizar el recurso y tener métodos alternativos de soporte ventilatorio, de ahí que se propone en

\section{Tabla 2. Stock prioritario fármacos}

\section{Cantidades disponibles conocidas de}

Antivirales, antimicrobianos y antifúngicos

Sedantes, analgésicos vasopresores, relajantes neuromusculares

Coloides, cristaloides

Hemoderivados; Banco de Sangre

Bloqueadores H2 Profilaxis de úlceras de stress y Heparinas: tromboembolismo venoso

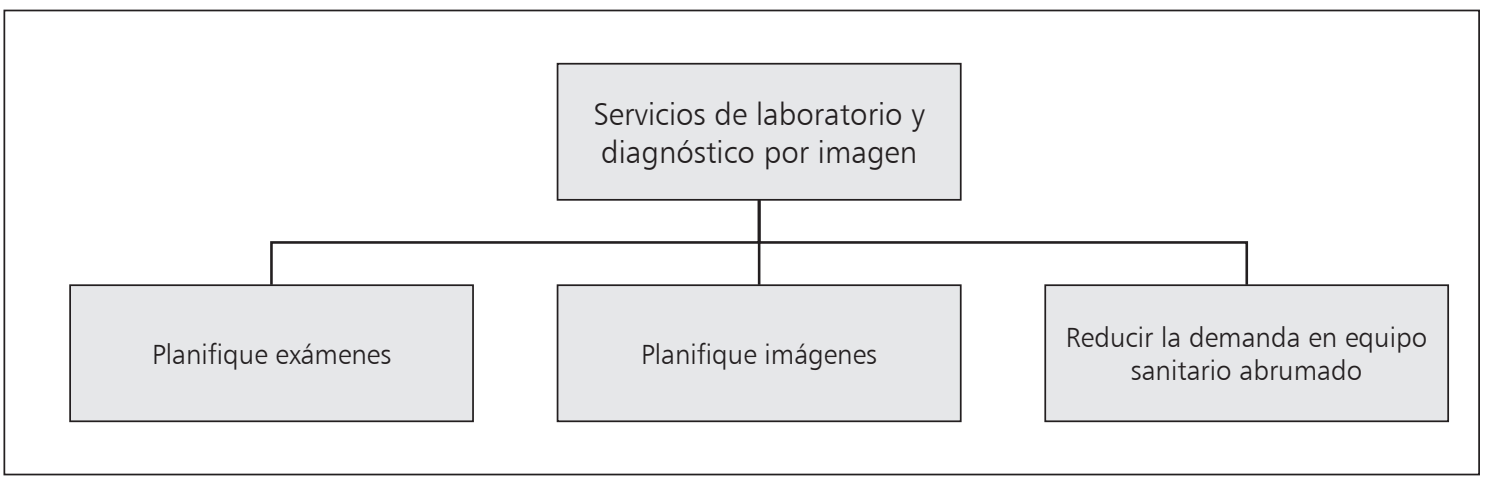

Figura 4. Optimización de tiempo; exámenes. 
forma gradual uso de máquinas de anestesia ${ }^{31}$, ventiladores pediátricos y de transporte, el empleo de cánula nasal de alto flujo y métodos no invasivos como CPAP, BiPAP ${ }^{23}$ (Figura 5). Existe riesgo de dispersión de partículas virales con el uso de ventilación mecánica no invasiva con respiradores de una rama. Esto por las fugas que se producen alrededor de la mascarilla, pero en casos seleccionado se puede emplear con el uso de filtros en rama inspiratoria, interface facial sin fuga, idealmente en un ambiente con presión negativa y sin duda con todos los elementos de protección personal.

Se han reconvertido ventiladores no invasivos utilizando el sistema de doble rama para permitir de esta forma disminuir el riesgo de contagio pero insistimos en las medidas de tratamiento pre UCI que pueden evitar admisiones.

Un aspecto no tan comentado pero radical en el funcionamiento de nuestras unidades de cuidados intensivos, en tiempos de pandemia, es el manejo de los pacientes críticos no COVID-19.
Idealmente, pudieran ser manejados en área diferentes. Se ha visto en el mundo un exceso de mortalidad no explicable por los casos de fallecimiento por COVID-1932,33. Esto probablemente es debido a que no han recibido la atención adecuada a su requerimiento en estos tiempos de crisis.

Un elemento que puede ser incluido en nuestro modelo de atención de expansión son los modelos de equipos de respuesta rápida ${ }^{34}$ (Figura 6), que recoge una serie de parámetros fisiológicos de los pacientes hospitalizados y cuando estos se alteran $\mathrm{y}$ dan un puntaje significativo sobre un umbral, alertan a los equipos de cuidado intensivo por la eventual complicación de estos pacientes; esto permite evaluarlos por el equipo de UCI, iniciar cuidados y trasladarlos en forma oportuna; así, de alguna se forma expanden los cuidados intensivo fuera de la UCI haciendo más eficiente los recursos previniendo complicaciones y admitiendo los enfermos forma más precoz antes que se compliquen.

Otro aspecto no menos importante son las

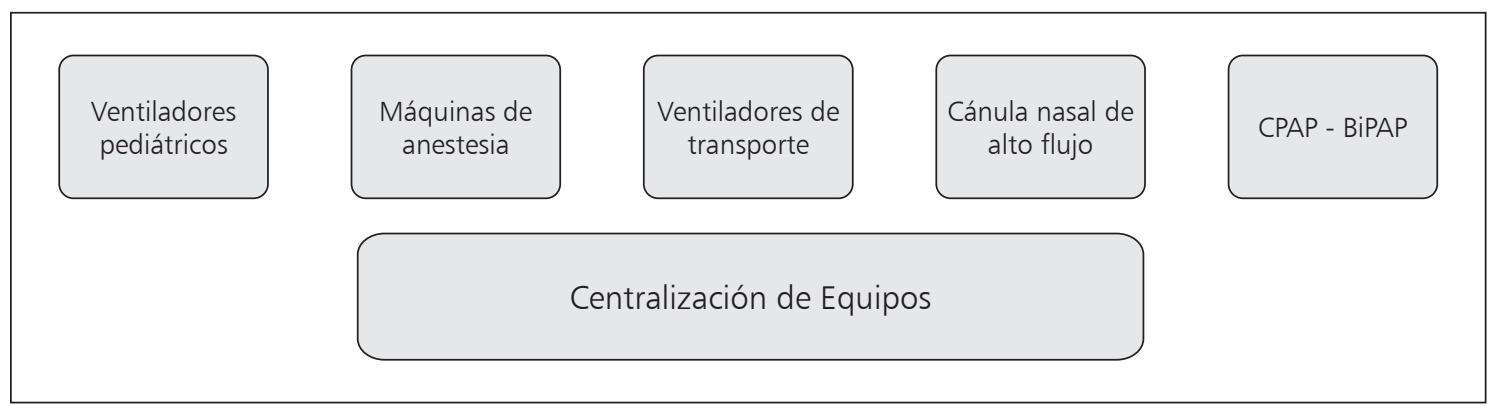

Figura 5. Alernativas en ventilación mecánica / Oxigenación.

\begin{tabular}{|c|c|c|c|c|c|c|c|}
\hline Categoría & 3 & 2 & 1 & 0 & 1 & 2 & 3 \\
\hline Frecuencia respiratoria & $\leq 8$ & & & $9-20$ & $21-30$ & $31-35$ & $\geq 36$ \\
\hline $\mathrm{SpO} 2(\%)$ & $<85$ & $85-89$ & $90-92$ & $\geq 93$ & & & \\
\hline Frecuencia cardíaca & $\leq 29$ & $30-39$ & $40-49$ & $50-99$ & 100-109 & $110-129$ & $\geq 130$ \\
\hline Presión arterial sistólica (mmHg) & $\leq 69$ & $70-79$ & $80-89$ & 100-199 & $\geq 200$ & & \\
\hline Temperatura $\left({ }^{\circ} \mathrm{C}\right)$ & $\leq 33,9$ & $34-34,9$ & $35-35,9$ & $36-37,9$ & $38-38,9$ & $\geq 39$ & \\
\hline Puntaje AVPU & & & & Vigil & $\begin{array}{c}\text { Reactivo a } \\
\text { estímulo } \\
\text { verbal }\end{array}$ & $\begin{array}{l}\text { Reactivo a } \\
\text { estímulo } \\
\text { nociceptivo }\end{array}$ & $\begin{array}{l}\text { Sin } \\
\text { respuesta }\end{array}$ \\
\hline
\end{tabular}

Figura 6. Parámetros de alerta. 
medidas de seguridad y accesos a nuestra UCI. Es necesario un control estricto del personal y visitantes mediante breve entrevista de síntomas y control temperatura al ingreso del hospital.

Por último, por la gran cantidad de pacientes que fallecen en los hospitales, se deben generar planes de contingencia ${ }^{35}$. Así, debemos tener planes para fallecimientos masivos para poder acomodar en forma temporal, segura y respetuosa el exceso de fallecidos, esto debe estar en congruencia con los aspectos sanitarios y legales.

En conclusión, esta pandemia ha estresado al máximo los requerimientos de cuidados críti- cos. Para poder atender la mayor cantidad de pacientes $y$ entregarles un cuidado oportuno, es necesario actuar de forma coordinada, premedita- da y eficaz, y desarrollar nuevas unidades capaces de entregar soporte vital. El trabajo integrado de diversos actores relevantes en el área hospitalaria permitirá diseñar estrategias innovadoras que permitan hacer frente a los desafíos que nos presenta esta situación.

Los autores manifiestan el más profundo reconocimiento a todo el personal de salud de nuestro país tanto de instituciones públicas, privadas, universitarias y de las Fuerzas Armadas y Carabineros, demostrando un alto grado de compromiso, enorme esfuerzo y excelencia en su trabajo ante esta situación tan adversa, dejando de lado muchas veces su familia y arriesgando su propia integridad por cuidar la vida de otros.

\section{Referencias}

1. Adhikari NK, Fowler RA, Bhagwanjee S, Rubenfeld GD. Critical care and the global burden of critical illness in adults. Lancet 2010; 376 (9749): 1339-46. doi: 10.1016/ S0140-6736(10)60446-1.

2. Prin $M$, Wunsch $H$. International comparisons of intensive care: informing outcomes and improving standards. Curr Opin Crit Care 2012; 18 (6): 700-6. doi:10.1097/ MCC.0b013e32835914d5.

3. Burki T. COVID-19 in Latin America. Lancet Infect Dis. 2020; 20 (5): 547-8. doi: 10.1016/S1473-3099(20)303030 .

4. Hossain T, Ghazipura M, Dichter JR. Intensive Care Role in Disaster Management Critical Care Clinics. Crit Care Clin 2019; 35 (4): 535-50. doi: 10.1016/j. ccc.2019.06.004.

5. Wax RS. Preparing the Intensive Care Unit for Disaster.
Crit Care Clin 2019; 35 (4): 551-62. doi: 10.1016/j. ccc.2019.06.008.

6. Simbana-Rivera K, Gómez-Barreno L, Guerrero J, Simbana-Guaycha F, Fernández R, López-Cortés A, et al. Interim Analysis of Pandemic Coronavirus Disease 2019 (COVID-19) and the SARS-CoV-2 virus in Latin America and the Caribbean: Morbidity, Mortality and Molecular Testing Trends in the Region. medRxiv [Internet]. 1 de enero de 2020;2020.04.25.20079863. Disponible en: http://medrxiv.org/content/early/2020/04/29/2020.04.25.20079863.abstract.

7. Sociedad Chilena de Medicina Intensiva. Encuesta nacional sobre ocupación de unidades criticas durante contingencia COVID19. 23 de mayo de 2020.

8. Guan W, Ni Z, Hu Y, Liang W, Ou C, He J, et al. Clinical Characteristics of Coronavirus Disease 2019 in China. N Engl J Med 2020; 382 (18): 1708-20. doi:10.1056/ NEJMoa2002032.

9. Zhou F, Yu T, Du R, Fan G, Liu Y, Liu Z, et al. Clinical course and risk factors for mortality of adult inpatients with COVID-19 in Wuhan, China: a retrospective cohort study [published correction appears in Lancet. 2020; 395 (10229): 1038] [published correction appears in Lancet. 2020; 395 (10229): 1038]. Lancet 2020; 395 (10229): 1054-62. doi: 10.1016/S01406736(20)30566-3.

10. Dawei Wang, Bo Hu, Chang Hu, Fangfang Zhu, Xing Liu, Jing Zhang, et al. Clinical Characteristics of 138 Hospitalized Patients With 2019 Novel Coronavirus-Infected Pneumonia in Wuhan, China. JAMA 2020; 323 (11): 1061-9. doi:10.1001/jama.2020.1585.

11. Yang X, Yu Y, Xu J, Shu H, Xia J, Liu H, et al. Clinical course and outcomes of critically ill patients with SARSCoV-2 pneumonia in Wuhan, China: a single-centered, retrospective, observational study [published correction appears in Lancet Respir Med. 2020; 8 (4): e26]. Lancet Respir Med 2020; 8 (5): 475-81. doi:10.1016/S22132600(20)30079-5.

12. Cummings M, Baldwin M, Abrams D, Jacobson S, Meyer B, Balough E, Aaron J, et al. Epidemiology, clinical course, and outcomes of critically ill adults with COVID-19 in New York City: a prospective cohort study [published online ahead of print, 2020 May 19]. Lancet 2020; S0140-6736 (20) 31189-2. doi:10.1016/S01406736(20)31189-2.

13. Wu C, Chen X, Cai Y, Xia J, Zhou X, Xu S, et al. Risk Factors Associated With Acute Respiratory Distress Syndrome and Death in Patients With Coronavirus Disease 2019 Pneumonia in Wuhan, China. JAMA Intern Med. Published online March 13, 2020. doi:10.1001/jamainternmed.2020.0994. 
14. Grasselli G, Zangrillo A, Zanella A, Antonelli M, Cabrini L, Castelli A, et al. Baseline Characteristics and Outcomes of 1591 Patients Infected With SARS-CoV-2 Admitted to ICUs of the Lombardy Region, Italy [published online ahead of print, 2020 Apr 6]. JAMA. 2020; 323 (16): 1574-81. doi:10.1001/jama.2020.5394.

15. Grasselli G, Pesenti A, Cecconi M. Critical Care Utilization for the COVID-19 Outbreak in Lombardy, Italy: Early Experience and Forecast During an Emergency Response. JAMA 2020; 323 (16): 1545-6. doi:10.1001/ jama.2020.4031.

16. Sandrock C. European Society of Intensive Care Medicine's Task Force for intensive care unit triage during an influenza epidemic or mass disaster. Chapter 4 . Manpower. Recommendations and standard operating procedures for intensive care unit and hospital preparations for an influenza epidemic or mass disaster. Intensive Care Med 2010; 36 Suppl 1: S32-7. doi: 10.1007/ s00134-010-1767-y.

17. Hick JL, Christian MD, Sprung CL. European Society of Intensive Care Medicine's Task Force for intensive care unit triage during an influenza epidemic or mass disaster. Chapter 2. Surge capacity and infrastructure considerations for mass critical care. Recommendations and standard operating procedures for intensive care unit and hospital preparations for an influenza epidemic or mass disaster. Intensive Care Med 2010; 36 Suppl 1: S11-S20. doi: 10.1007/s00134-010-1761-4.

18. Litton E, Bucci T, Chavan S, Ho Y, Holley A, Howard $G$, et al. Surge capacity of intensive care units in case of acute increase in demand caused by COVID-19 in Australia [published online ahead of print, $2020 \mathrm{Apr}$ 19]. Med J Aust. 2020;10.5694/mja2.50596. doi:10.5694/ mja2.50596.

19. Goh KJ, Wong J, Claudia Tien JC, Ng SY, Wen SD, Phua GC, et al. Preparing your intensive care unit for the COVID-19 pandemic: practical considerations and strategies. Crit Care 2020; 24 (1): 215. Published 2020 May 11. doi:10.1186/s13054-020-02916-4.

20. Hick J, Einav S, Hanfling D, Kissoon N, Dichter J, Devereaux A, et al. Surge capacity principles: care of the critically ill and injured during pandemics and disasters: CHEST consensus statement. Chest 2014; 146 (4 Suppl): e1S-e16S. doi: 10.1378/chest.14-0733.

21. Joynt G, Loo S, Taylor B, Margalit G, Christian M, Sandrock C, et al. Chapter 3. Coordination and collaboration with interface units. Recommendations and standard operating procedures for intensive care unit and hospital preparations for an influenza epidemic or mass disaster. Intensive Care Med 2010; 36 Suppl 1 (Suppl 1): S21-S31. doi:10.1007/s00134-010-1762-3.
22. Richards GA, Sprung CL. European Society of Intensive Care Medicine's Task Force for intensive care unit triage during an influenza epidemic or mass disaster. Chapter 9. Educational process. Recommendations and standard operating procedures for intensive care unit and hospital preparations for an influenza epidemic or mass disaster. Intensive Care Med 2010; 36 Suppl 1(Suppl 1): S70-S79. doi: 10.1007/s00134-010-1768-X.

23. Aziz S, Arabi YM, Alhazzani W, Evans L, Citerio G, Fischkoff K, et al. Managing ICU surge during the COVID-19 crisis: Rapid Guidelines. Intensive Care Med. 2020;1-23. DOI: 10.1007/s00134-020-06092-5.

24. Einav S, Hick JL, Hanfling D, et al. Surge capacity logistics: care of the critically ill and injured during pandemics and disasters: CHEST consensus statement. Chest. 2014; 146 (4 Suppl): e17S-43S. doi:10.1378/ chest.14-0734.

25. Debergh DP, Myny D, Van Herzeele I, Van Maele G, Reis Miranda D, Colardyn F. Measuring the nursing workload per shift in the ICU. Intensive Care Med. 2012; 38 (9): 1438-44. doi:10.1007/s00134-012-2648-3.

26. Miranda DR, Nap R, de Rijk A, Schaufeli W, Iapichino G. TISS Working Group. Therapeutic Intervention Scoring System. Nursing activities score. Crit Care Med 2003; 31 (2): 374-82. doi:10.1097/01. CCM.0000045567.78801.CC.

27. Tran K, Cimon K, Severn M, Pessoa-Silva CL, Conly J. Aerosol generating procedures and risk of transmission of acute respiratory infections to healthcare workers: a systematic review. PLoS One 2012; 7 (4): e35797. doi: 10.1371/journal.pone.0035797.

28. Centers for Disease Control and Prevention. Interim Infection Prevention and Control Recommendations for Patients with Suspected or Confirmed Coronavirus Disease 2019 (COVID-19) in Healthcare Settings. Update May 18, 2020. Accessed May 24, 2020. https://www.cdc. gov/coronavirus/2019-ncov/hcp/infection-control-recommendations.html.

29. Sprung CL, Kesecioglu J. European Society of Intensive Care Medicine's Task Force for intensive care unit triage during an influenza epidemic or mass disaster. Chapter 5. Essential equipment, pharmaceuticals and supplies. Recommendations and standard operating procedures for intensive care unit and hospital preparations for an influenza epidemic or mass disaster. Intensive Care Med. 2010; 36 Suppl 1: S38-S44. doi: 10.1007/s00134010-1763-2.

30. Ranney ML, Griffeth V, Jha AK. Critical Supply Shortages - The Need for Ventilators and Personal Protective Equipment during the Covid-19 Pandemic. N Engl J Med. 2020;382(18):e41. doi:10.1056/NEJMp2006141. 
31. Hick JL, Rubinson L, O'Laughlin DT, Farmer JC. Clinical review: allocating ventilators during large-scale disasters-problems, planning, and process. Crit Care 2007; 11 (3): 217. doi:10.1186/cc5929.

32. New York City Department of Health and Mental Hygiene (DOHMH) COVID-19 Response Team. Preliminary Estimate of Excess Mortality During the COVID-19 Outbreak-New York City, March 11-May 2, 2020. MMWR Morb Mortal Wkly Rep. 2020; 69 (19): 603-5. Published 2020 May 15. doi: 10.15585/mmwr. mm6919e5.

33. Nogueira PJ, Nobre MA, Nicola PJ, Furtado C, Vaz
Carneiro A. Excess Mortality Estimation During the COVID-19 Pandemic: Preliminary Data from Portugal [published online ahead of print, 2020 Apr 27]. Acta Med Port 2020; 10.20344/amp.13928. doi:10.20344/ amp. 13928.

34. Lyons PG, Edelson DP, Churpek MM. Rapid response systems. Resuscitation 2018; 128: 191-7. doi:10.1016/j. resuscitation.2018.05.013.

35. WHO Interm Guidance. Infection Prevention and Control for the safe management of a dead body in the context of COVID-19. J Hosp Infect 2020; 104 (3): 24651. 